
\title{
25 Research Soure \\ Differences in Gene Expression Between High- and Low-Grade Serous Ovarian Cancers: Implications for Diagnosis and Prognosis
}

Jianhao Xu

Affiliated Kunshan Hospital of Jiangsu University

Qian Wang

Medical College of Soochow University

Fang Cao

Affiliated Kunshan Hospital of Jiangsu University

Zhiyong Deng

Affiliated Kunshan Hospital of Jiangsu University

Xiaojiao Gao

Affiliated Kunshan Hospital of Jiangsu University

Tingting Gu

Affiliated Kunshan Hospital of Jiangsu University

Tingting Liu

Affiliated Kunshan Hospital of Jiangsu University

Song Xu ( $\nabla$ xs19780116@163.com)

Affiliated Kunshan Hospital of Jiangsu University

Wenjuan Gan

Dushu Lake Hospital Affiliated to Soochow University

\section{Research Article}

Keywords: high-grade serous ovarian cancer, low-grade serous ovarian cancer, diagnosis, prognosis, differentially expressed genes

Posted Date: February 15th, 2022

DOI: https://doi.org/10.21203/rs.3.rs-963632/v3

License: (c) (1) This work is licensed under a Creative Commons Attribution 4.0 International License.

Read Full License 


\section{Abstract}

Background: The clinical presentations of high-grade serous ovarian cancer (HGSOC) and low-grade serous ovarian cancer (LGSOC) differ. In this study, we aimed to identify the essential molecules for the diagnosis and prognosis of these $\mathrm{OC}$ subtypes.

Methods: Differentially expressed genes between HGSOC and LGSOC were identified using three GEO series. The functional enrichment analysis was performed to investigate different biological processes and pathways. The protein-protein interaction network was constructed, and the discovered hub genes were frequently validated using prognostic correlation and immunohistochemistry (IHC) in GEPIA and HPA databases. Finally, we screened out BIRC5 and used IHC to detect its expression in 20 cases of borderline serous tumors, 20 cases of LGSOC, and 38 cases of HGSOC, and further analyzed its correlation with clinical characteristics.

Results: In comparison with LGSOC, 79 upregulated and 85 downregulated genes were identified in HGSOC. The biological roles of these genes were mainly centered on the cell cycle process and chromosomal segregation. Among the 10 hub genes chosen, BIRC5 is positively related to the overall survival of patients with $\mathrm{OC}(\mathrm{p}=0.014)$ and can distinguish $\mathrm{OC}$ from normal ovarian tissue. In addition to database analysis, we verify BIRC5 through the specimen resources in our case database. According to the IHC results of our specimens, we found that BIRC5 can not only distinguish HGSOC and LGSOC but also positively correlate with the age, preoperative CA125 level, FIGO stage『and TP53/Ki-67 expression in tumor specimens.

Conclusions: BIRC5 is a reliable marker that can distinguish HGSOC from LGSOC, guide prognosis, and be utilized in clinical IHC.

\section{Background}

Serous carcinoma, mucinous carcinoma, endometrioid carcinoma, and clear cell carcinoma are the histological subtypes of epithelial ovarian cancer (OC)(1).The most frequent subtype, Serous OC is further subdivided into high-grade and low-grade serous OCs (abbreviated as HGSOC and LGSOC, respectively)(2). The clinical characteristics of the two serous OCs differ(2). For example, the data set GSE151335 was used to verify the differences in progress-free survival (PFS) and OS between HGSOC and LGSOC. The platform for GSE151335 is GPL28589 [Oxford Classifier of Carcinoma of the Ovary]. Patients with HGSOC exhibited worse PFS ( $p=0.011$, Figure 1A) and OS $(p=0.0027$, Figure 1B).

HGSOC is now linked with TP53 mutations, whereas LGSOC is associated with BRAF and KRAS mutations as the benign/borderline adenoma progression( $(3,4)$. Given that BRAF and KRAS mutations are often detected through next-generation sequencing(5), time and economical expenses associated with LGSOC diagnosis are high. Immunohistochemistry (IHC) techniques are more commonly utilized for differential diagnosis in real-world clinical settings. TP53 is a precise marker for the differential diagnosis of HGSOC(6), although it occasionally produces errors in clinical practice. Furthermore, the Gene 
Expression Profiling Interactive Analysis (GEPIA) website indicates that TP53 has little predictive value for the prognosis of $O C$ patients( $\mathrm{pHR}=0.93$, Figure 2). Thus, the principal clinical objective of this research is to identify critical molecules that can distinguish diagnosis and inform prognosis based on HGSOC and LGSOC expression differences.

Therefore, the primary clinical goal of this research is to screen critical molecules that can differentiate diagnosis and guide prognosis based on differential genes expression of HGSOC and LGSOC.

\section{Methods}

\subsection{Microarray data}

Gene Expression Omnibus (GEO, http://www.ncbi.nlm.nih.gov/geo) functions as a public functional genomics database of high throughput gene expression data, chips, and microarrays(7). Microarray data of the human $\mathrm{OC}$ dataset were screened through the GEO database. The search strategy was as follows: ("ovarian neoplasms"[MeSH Terms] OR ovarian cancer[All Fields]) AND "Homo sapiens"[porgn] AND ("gse" [Filter] AND "Expression profiling by array"[Filter] AND "attribute name tissue"[Filter]). Three gene expression profiles in HGSOC and LGSOC tissues were screened and downloaded from a total of 136 GEO Series (GSEs), namely GSE73638, GSE27651 and GSE14001. Platforms for GSE73638 is GPL20967 [ADXOCv1a520630] Almac OC Disease Specific Array, while for GSE27651 and GSE14001 is GPL570[HGU133_Plus_2] Affymetrix Human Genome U133 Plus 2.0 Array. GSE73638 contains 13 HGSOC samples and 7 LGSOC samples. GSE27651 contains 21 HGSOC samples and 13 LGSOC samples. GSE14001 contains 10 HGSOC samples and 10 LGSOC samples.

\subsection{Identification of DEGs}

GEO2R is regarded as an interactive online tool that has been designed to compare two or more datasets in the GEO series to identify differentially expressed genes (DEGs)(7). DEGs between HGSOC and LGSOC tissues were identified using GEO2R, with the cut-off points of adj.p $<0.05$ and $|\log F C|>1$. DEGs in GSE14001 were identified only with the threshold of adj.p $<0.05$ due to the limited DEGs by the threshold above to ensure sufficient DEGs for subsequent analyses. Therefore, we first detected the intersection DEGs between GSE73638 and GSE27651 and then used GSE14001 for a second confirmation. We used online Wien software to detect the intersection DEGs between the datasets. Volcano and the heat map were painted to picture these DEGs using the R ggplot2 and heat map package.

\subsection{Function enrichment analysis}

The Gene Ontology (GO) datasets and Kyoto Encyclopedia of Gene and Genome (KEGG) pathway enrichment were used to analyze DEGs at the functional level with WEB-based Gene Set Analysis Toolkit (WebGestalt, http://www.webgestalt.org/option.php, version 2019). WebGestalt is a functional enrichment analysis web tool, which has on average 26,000 unique users from 144 countries and territories per year, according to Google Analytics(8). The results were pictured in the R ggplot2 package. $\mathrm{P}<0.05$ was considered statistically significant. 


\subsection{GSEA analysis}

Gene Set Enrichment Analysis (GSEA) is a computational method that determines whether an a priori defined set of genes shows statistically significant, concordant differences between two biological states (e.g. HGSOC and LGSOC)(9). The GSEA approach was applied to reconfirm function enrichment analysis in GSEA software version 4.1.0, which uses predefined gene sets from the Molecular Signatures Database (MSigDB v7.4).

\subsection{PPI network construction and module analysis}

Search Tool for the Retrieval of Interacting Genes (STRING, http://string-db.org, version 11.5) is a database of known and predicted protein-protein interactions (PPI), which was applied to visualize the potential gene interaction network(10). The minimum required interaction score is 0.700 , which is high confidence. Cytoscape (a public bioinformatics software, https://cytoscape.org/, version 3.8.2) is an open-source software platform for visualizing complex networks and integrating these with any attribute data(11). The plug-in Molecular Complex Detection (MCODE, version 2.0.0) app of Cytoscape clusters a given network based on the topology to find densely connected regions with the following criteria: degree cutoff $=2$, node score cutoff $=0.2$, K-Core $=2$, Max. Depth $=100(12)$.

\subsection{Hub genes selection and analysis}

Another plug-in app cytoHubba (version 0.1 ) of Cytoscape predicts and explores important nodes and sub-networks in a given network by different topological algorithms(13). In the present study, the top nodes were ranked by Radiality algorithms. The OS and stage analysis of 10 hub genes in OC was conducted by GEPIA (http://gepia.cancer-pku.cn/index.html) database(14). The protein level was verified using OC pathological tissue slices in Human Protein Atlas (HPA, https://www.proteinatlas.org/, Version: 20.1) database(15).

\subsection{Samples and histologic assessment}

From January 2014 to September 2021, 20 borderline serous tumors(BST) cases, 20 LGSOC cases, and HGSOC 38 cases with complete clinicopathological data were obtained from the Department of Pathology, Kunshan Hospital, Jiangsu University. All patients have not received previous adjuvant systemic therapy. All tumors archived in the pathology department were pathologically diagnosed according to the World Health Organization standards (2014) and were staged by the International Federation of Obstetrics and Gynecology (FIGO). 2 attending pathologists independently reviewed the tissue sections, and the difference was reviewed by the third deputy chief physician/chief physician.

\subsection{Immunohistochemistry}

All specimens were fixed with paraformaldehyde, embedded in paraffin, and prepared as $4 \mu \mathrm{m}$ thick serial sections. Paraffin sections were processed following the GTVisionTM $\otimes$ Detection System(Gene Tech company, Item No.: GK5007). The primary antibody was the BIRC5 antibody(Gene Tech company, Clone No.: EP2880Y). The results were analyzed by the double-blind method. According to the HPA database, 
BIRC5 immunoreactivity was localized in the nucleus/cytoplasm. Positive staining of BIRC5 was scored using the Allred score system(16). The Allred score was calculated by adding the percentage of positive tumor cells $(0$, none; $1,<1 \% ; 2,1-10 \% ; 3,11-33 \% ; 4,34-66 \%$; and $5,67-100 \%)$ and the average intensity of immunoreactivity ( 0 , no staining; 1 , weak; 2 , moderate; and 3 , strong), with a range from 0 to 8 .

\section{Results}

\subsection{Identification of DEGs between HGSOC and LGSOC}

Among the 136 GSEs screened, we evaluated a total of four series that comprised HGSOC and LGSOC broad gene expression microarray data, namely GSE73638, GSE73551, GSE27651, and GSE14001. Because the series GSE73638 and GSE73551 belong to the same study, the current study included only GSE73638 because it had a larger sample size. Table 1 summarizes the essential information for the three GSEs.

Table 1

Sample details of the selected GEO Series.

\begin{tabular}{|lllllll|}
\hline $\begin{array}{l}\text { GEO } \\
\text { Series }\end{array}$ & $\begin{array}{l}\text { Publication } \\
\text { date }\end{array}$ & Platform & Samples & Source & Cell type & Case \\
number
\end{tabular}


We utilized the GEO2R online program to identify 1465, 9914, and 230 distinct genes from GSE73638, GSE27651, and GSE14001, respectively (Figure 3A, adj.P $₫ 0.01$, $|\log F C|>1$ ). The DEGs in the GSE14001 were restricted by the threshold above. 9500 DEGs in GSE14001 were identified with a threshold adj.P $₫ 0.05$ and utilized to confirm the overlapping DEGs between GSE73638 and GSE27651. According to the Venn diagram, overlapping DEGs between GSE73638 and GSE27651 comprised 157 upregulated and 204 downregulated genes (Figure 3B). Following validation by GSE14001, 79 upregulated and 85 downregulated genes were chosen for the current study (Figure 3C). We used heat maps to depict the distribution of screened gene expression in each GSE between HGSOC and LGSOC (Figure 3D).

\subsection{Enrichment analysis for DEGs}

We used the WebGestalt web tool to perform GO and KEGG enrichment analyses to identify the most important biological processes (BPs) and pathways. In total, 164 DEGs were primarily enriched in the BPs associated with mitotic cell cycle, organelle fission, and nuclear division (Figure $4 A$ ) and pathways such as hepatitis C, micro RNAs in cancer, and chronic myeloid leukemia (Figure 4B). In addition, we used GSEA to validate the GOBP, which was substantially represented with a normalized p-value of $<0.05$. In the HGSOC group, 363, 8, and 31 BPs were enriched in GSE73638, GSE27651, and GSE14001, respectively, and three BPs were enriched in all three GSEs (Figure 4C). Meiotic cell cycle process, homologous chromosome segregation, and meiosis I cell cycle process were the BPs confirmed by the three GSEs (Figure 4D).

\subsection{PPI network construction and significant module identification}

We utilized the String database to estimate the protein-level connection of the overlapped DEGs (Figure 5A). We improved the visualization with Cytoscape software and constructed a PPI network with 115 nodes and 894 edges (Figure 5B). MCODE was used to divide the PPI network into four modules (Figure 5C); the first module had 38 nodes and 661 edges (MCODE score 35.730), the second module had 7 nodes and 21 edges (MCODE score 7.000), the third module had 5 nodes and 10 edges (MCODE score 5.000), and the fourth module had 3 nodes and 3 edges (MCODE score 3.000). We utilized Cytohubba to filter the top 10 Hubba nodes, namely BIRC5, CDC20, CDK1, CDKN3, MKI67, NUSAP1, RRM2, TOP2A, TPX2, and UBE2C, for additional investigation using Radiality topological techniques (Figure 5D).

\subsection{Analysis of the hub genes}

We used the GEPIA database to investigate the connection between the 10 hub genes and OC $\mathrm{OS} /$ staging. Among the 10 hub genes, only BIRC5 was found to be favorably linked with OS in OC ( $\mathrm{pHR}=$ 0.014 , Figure $6 \mathrm{~A})$, and only RRM2 was found to be negatively correlated with OC staging $(p=0.0251$, Figure 6B).

Based on the results above, BIRC5 and RRM2 with potential therapeutic utility were chosen from a list of 10 hub genes. Then, we predict the ability of BIRC5 and RRM2 to distinguish between normal ovarian tissue and OC by the HPA database. Using antibodies HPA002830 and CAB004270, we found that the 
expression of BIRC5 in OC tissues was greater than that in normal ovarian tissues (Figure 7A-C). RRM2 expression in OC tissues could not be identified with HPA056994 in both OC and normal ovarian tissues (Figure 7D).

\subsection{Verify genes with potential clinical value using our cases}

We analyzed the correlation between the BIRC5 IHC staining score and clinicopathological parameters (Table 2). We included three different pathological types of ovarian tumors: BST, LGSOC, and HGSOC. Through ANOVA test, we found that in addition to pathological types $(p<0.0001)$, age $(p<0.0001)$, preoperative CA125 level $(p=0.0175)$, FIGO stage $(p=0.0079)$, TP53 $(p<0.0001)$ and Ki67 expression ( $p$ $<0.0001)$ are also related to BIRC5 expression. 
Table 2

Relationships between BIRC5 staining score and clinicopathological parameters.

\begin{tabular}{|c|c|c|c|c|}
\hline Pathological characteristics & Cases(n) & BIRC5 Scores & $P$ value & $F$ \\
\hline Total & 78 & $1.84 \pm 2.15$ & & \\
\hline Age & & & $<0.0001$ & 10.75 \\
\hline$<37$ & 19 & $0.42 \pm 0.82$ & & \\
\hline$[37,49)$ & 19 & $1.15 \pm 1.63$ & & \\
\hline$[49,61)$ & 20 & $2.80 \pm 2.27$ & & \\
\hline$\geq 61$ & 20 & $3.36 \pm 2.05$ & & \\
\hline CA125 & & & 0.0175 & 3.612 \\
\hline$<39.98$ & 18 & $2.06 \pm 2.34$ & & \\
\hline$[39.98,126.80)$ & 17 & $1.29 \pm 2.08$ & & \\
\hline$[126.80,618.38)$ & 19 & $1.58 \pm 1.53$ & & \\
\hline$\geq 618.38$ & 17 & $3.39 \pm 2.00$ & & \\
\hline FIGO & & & 0.0079 & 5.295 \\
\hline Q & 18 & $1.17 \pm 2.01$ & & \\
\hline प & 10 & $3.00 \pm 2.14$ & & \\
\hline 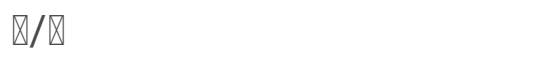 & 30 & $3.10 \pm 1.97$ & & \\
\hline Allred score of TP53 & & & $<0.0001$ & 10.33 \\
\hline$<3$ & 10 & $1.20 \pm 1.66$ & & \\
\hline$[3,5)$ & 9 & $0.44 \pm 1.26$ & & \\
\hline$[5,7)$ & 22 & $0.82 \pm 1.15$ & & \\
\hline$\geq 7$ & 37 & $3.19 \pm 2.22$ & & \\
\hline $\mathrm{Ki}-67(+) \%$ & & & $<0.0001$ & 20.52 \\
\hline$<5$ & 14 & $0.50 \pm 1.35$ & & \\
\hline$[5,30)$ & 28 & $0.71 \pm 1.19$ & & \\
\hline$[30,70)$ & 17 & $2.76 \pm 1.86$ & & \\
\hline$\geq 70$ & 19 & $4.00 \pm 1.95$ & & \\
\hline
\end{tabular}

FIGO, International Federation of Gynecology and Obstetrics; BST, borderline serous tumors; LGSOC, low-grade serous ovarian cancer; HGSOC, high-grade serous ovarian cancer. Positive staining of BIRC5 was scored using the Allred score system. 


\begin{tabular}{|lcccc|}
\hline Pathological characteristics & Cases(n) & BIRC5 Scores & P value & F \\
\hline Pathology type & & & $<0.0001$ & 45.45 \\
\hline BST & 20 & $0.30 \pm 0.64$ & \\
LGSOC & 20 & $0.45 \pm 0.86$ & \\
\hline HGSOC & 38 & $3.55 \pm 1.92$ & \\
\hline $\begin{array}{l}\text { FIGO, International Federation of Gynecology and Obstetrics; BST, borderline serous tumors; LGSOC, } \\
\text { low-grade serous ovarian cancer; HGSOC, high-grade serous ovarian cancer. Positive staining of } \\
\text { BIRC5 was scored using the Allred score system. }\end{array}$ \\
\hline
\end{tabular}

We further analyzed the correlation between BIRC5 expression and various clinicopathological parameters. Figure 8A shows the proportion of high BIRC5 expression in different clinicopathological parameters groups, and Figure 8B shows the statistical correlation between BIRC5 expression and different clinicopathological parameters. Age $(p<0.0001)$, preoperative CA125 level $(p=0.0348)$, FIGO stage $(p<0.0001)$, TP53/Ki67 expression level $(p<0.0001)$ and the malignant degree of OC $(p<0.0001)$ are positively correlated with BIRC5 expression.

\section{Discussion}

HGSOC and LGSOC have diverse clinical presentations, and the prognosis differs even within the same group. However, our literature survey indicated that only a few studies had compared HGSOC with LGSOC. Thus, the goal of the present study was to identify and validate the essential molecules that can help distinguish diagnoses and guide prognoses of the two OC subtypes.

We identified 164 robust DEGs by using the three GSEs. Based on WebGesalt and GSEA analysis, we discovered that the GOBPs differing between HGSOC and LGSOC are mainly the cell cycle process and chromosomal segregation, which is compatible with HGSOC's proliferative activity $(17,18)$. We then selected 10 hub genes out of 164 DEGs. We found that only BIRC5 is favorably linked with OS, and only RRM2 is inversely correlated with staging, indicating these two genes' therapeutic significance.

Furthermore, as stated in the introduction that the chosen molecules should be used in clinical IHC, we used the HPA website to assess the feasibility of BIRC5 and RRM2 as IHC markers. The findings indicated that BIRC5 could differentiate between normal and OC tissues; however, RRM2 could not be detected. The core of this article is to explore the IHC indicators for distinguishing HGSOC and LGSOC. Therefore, for BIRC5, in addition to predicting its ability to distinguish benign and malignant through the HPA database, we also need to verify its ability to distinguish HGSOC from LGSOC through our pathological specimens. We collected 20 BST, 20 LGSOC, and 38 HGSOC specimens. IHC experiments found that BIRC 5 can distinguish HGSOC and LGSOC and positively correlate with the patients' age, preoperative CA125 level, FIGO stage, TP53, and Ki67 expression. In conclusion, BIRC5 offers a high clinical application value.

BIRC5 is an inhibitor of the apoptosis gene family that encodes harmful regulatory proteins, which suppress apoptotic cell death. BIRC5 expression is high during fetal development and in most cancers 
but low in adult tissues(19). Yin et al. used bioinformatics tools to investigate the OC OVDM1 cell line and confirmed the clinical significance of BIRC5, which is consistent with the findings of our study(20). A meta-analysis by $\mathrm{He} \mathrm{X}$ et al. indicated that the protein survivin is closely linked to FIGO staging and tumor grade of $\mathrm{OC}(21)$. It is also worth mentioning that for more than 20 years, BIRC5 has been regarded as a cancer treatment target(22).For example, Ozreti et al. revealed that the Hedgehog signaling pathway is linked to OC pathogenesis and that BIRC5 might be a novel pathway target(23). Wang et al. utilized an orthotropic OC mouse model to confirm that miR-203 suppresses ovarian tumor metastasis by targeting BIRC5 to prevent EMT(24). In this study, we found that the high expression of BIRC5 is closely related to the high level of CA125, the advanced FIGO stage, and the malignancy of OC. From the above, we know that BIRC5 regulates tumor cell proliferation. It suggests that BIRC5 can assist in judging the prognosis of patients with ovarian cancer and may serve as a tumor treatment target.

The present study also has some limitations. The selection criteria for DEGs in GSE14001 are only specified as adj.p $<0.05$, which is utilized as a secondary verification for the summary results of DEGs in the other two datasets.

\section{Conclusion}

BIRC5 is the crucial molecule identified in this investigation that can distinguish diagnosis, guide prognosis, and be applied in clinical settings. The importance of this study is to provide a critical marker to guide clinical practice.

\section{Declarations}

\subsection{Ethics approval and consent to participate}

Not applicable.

\subsection{Consent for publication}

Not applicable.

\subsection{Availability of data and materials}

The datasets used and/or analyzed during the current study are available from the corresponding author on reasonable request.

\subsection{Competing interests}

The authors declare that they have no competing interests.

\subsection{Funding}


The current study was supported by grants from the 2019 Kunshan Key R\&D Plan (Ecological Agriculture and Social Development) -Social Development Science and Technology Project (KS1941).

\subsection{Authors' contributions}

Conceptualization: XS, GWJ; Database search: XJH, WQ, CF; Data analysis: XJH, GXJ; Original draft writing: XJH, WQ, GTT; Review, editing, and final approval: DZY, LTT; Research supervision: XS, GWJ, DZY.

\subsection{Acknowledgements}

Not applicable.

\section{References}

1. Kaku T, Ogawa S, Kawano Y, Ohishi Y, Kobayashi H, Hirakawa T, et al. Histological classification of ovarian cancer. Medical electron microscopy : official journal of the Clinical Electron Microscopy Society of Japan. 2003;36(1):9-17.

2. Goulding EA, Simcock B, McLachlan J, van der Griend R, Sykes P. Low-grade serous ovarian carcinoma: A comprehensive literature review. The Australian \& New Zealand journal of obstetrics \& gynaecology. 2020;60(1):27-33.

3. Ramalingam P. Morphologic, Immunophenotypic, and Molecular Features of Epithelial Ovarian Cancer. Oncology (Williston Park, NY). 2016;30(2):166-76.

4. Kurman RJ. Origin and molecular pathogenesis of ovarian high-grade serous carcinoma. Annals of oncology : official journal of the European Society for Medical Oncology. 2013;24 Suppl 10:x16-21.

5. Surrey LF, Oakley FD, Merker JD, Long TA, Vasalos P, Moncur JT, et al. Next-Generation Sequencing (NGS) Methods Show Superior or Equivalent Performance to Non-NGS Methods on BRAF, EGFR, and KRAS Proficiency Testing Samples. Archives of pathology \& laboratory medicine. 2019;143(8):980-4.

6. Köbel M, Piskorz AM, Lee S, Lui S, LePage C, Marass F, et al. Optimized p53 immunohistochemistry is an accurate predictor of TP53 mutation in ovarian carcinoma. The journal of pathology Clinical research. 2016;2(4):247-58.

7. Barrett T, Wilhite SE, Ledoux P, Evangelista C, Kim IF, Tomashevsky M, et al. NCBI GEO: archive for functional genomics data sets-update. Nucleic acids research. 2013;41(Database issue):D991-5.

8. Liao Y, Wang J, Jaehnig EJ, Shi Z, Zhang B. WebGestalt 2019: gene set analysis toolkit with revamped Uls and APIs. Nucleic acids research. 2019;47(W1):W199-w205.

9. Subramanian A, Tamayo P, Mootha VK, Mukherjee S, Ebert BL, Gillette MA, et al. Gene set enrichment analysis: a knowledge-based approach for interpreting genome-wide expression profiles. Proceedings of the National Academy of Sciences of the United States of America. 2005;102(43):15545-50.

10. Szklarczyk D, Franceschini A, Wyder S, Forslund K, Heller D, Huerta-Cepas J, et al. STRING v10: protein-protein interaction networks, integrated over the tree of life. Nucleic acids research. 
2015;43(Database issue):D447-52.

11. Shannon P, Markiel A, Ozier O, Baliga NS, Wang JT, Ramage D, et al. Cytoscape: a software environment for integrated models of biomolecular interaction networks. Genome research. 2003;13(11):2498-504.

12. Bandettini WP, Kellman P, Mancini C, Booker OJ, Vasu S, Leung SW, et al. MultiContrast Delayed Enhancement (MCODE) improves detection of subendocardial myocardial infarction by late gadolinium enhancement cardiovascular magnetic resonance: a clinical validation study. Journal of cardiovascular magnetic resonance : official journal of the Society for Cardiovascular Magnetic Resonance. 2012;14(1):83.

13. Chin $\mathrm{CH}$, Chen $\mathrm{SH}, \mathrm{Wu} \mathrm{HH}, \mathrm{Ho} \mathrm{CW}$, Ko MT, Lin CY. cytoHubba: identifying hub objects and subnetworks from complex interactome. BMC systems biology. 2014;8 Suppl 4(Suppl 4):S11.

14. Tang Z, Li C, Kang B, Gao G, Li C, Zhang Z. GEPIA: a web server for cancer and normal gene expression profiling and interactive analyses. Nucleic acids research. 2017;45(W1):W98-w102.

15. Uhlén M, Fagerberg L, Hallström BM, Lindskog C, Oksvold P, Mardinoglu A, et al. Proteomics. Tissuebased map of the human proteome. Science (New York, NY). 2015;347(6220):1260419.

16. Harvey JM, Clark GM, Osborne CK, Allred DC. Estrogen receptor status by immunohistochemistry is superior to the ligand-binding assay for predicting response to adjuvant endocrine therapy in breast cancer. Journal of clinical oncology : official journal of the American Society of Clinical Oncology. 1999;17(5):1474-81.

17. Cunningham JM, Vierkant RA, Sellers TA, Phelan C, Rider DN, Liebow M, et al. Cell cycle genes and ovarian cancer susceptibility: a tagSNP analysis. British journal of cancer. 2009;101(8):1461-8.

18. Tanaka K, Hirota T. Chromosome segregation machinery and cancer. Cancer science. 2009;100(7):1158-65.

19. Wheatley SP, Altieri DC. Survivin at a glance. Journal of cell science. 2019;132(7).

20. Yin S, Du J, Zhang J, Zhang X, Ma K. Identification of Key Genes and Pathway for Ovarian Neoplasms Using the OVDM1 Cell Line Based on Bioinformatics Analysis. Medical science monitor: international medical journal of experimental and clinical research. 2019;25:4305-13.

21. He X, Yang K, Wang H, Chen X, Wu H, Yao L, et al. Expression and clinical significance of survivin in ovarian cancer: A meta-analysis. PloS one. 2018;13(5):e0194463.

22. Li F, Aljahdali I, Ling X. Cancer therapeutics using survivin BIRC5 as a target: what can we do after over two decades of study? Journal of experimental \& clinical cancer research : CR. 2019;38(1):368.

23. Ozretić P, Trnski D, Musani V, Maurac I, Kalafatić D, Orešković S, et al. Non-canonical Hedgehog signaling activation in ovarian borderline tumors and ovarian carcinomas. International journal of oncology. 2017;51(6):1869-77.

24. Wang B, Li X, Zhao G, Yan H, Dong P, Watari H, et al. miR-203 inhibits ovarian tumor metastasis by targeting BIRC5 and attenuating the TGF $\beta$ pathway. Journal of experimental \& clinical cancer research : CR. 2018;37(1):235. 
Figures

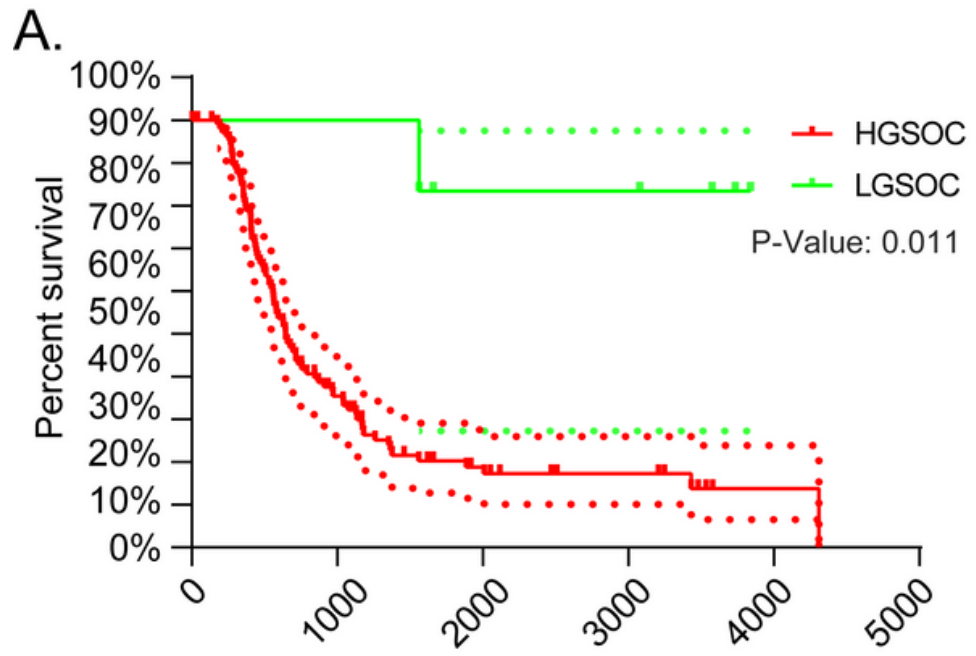

Progress-free Survival (Days)
B.

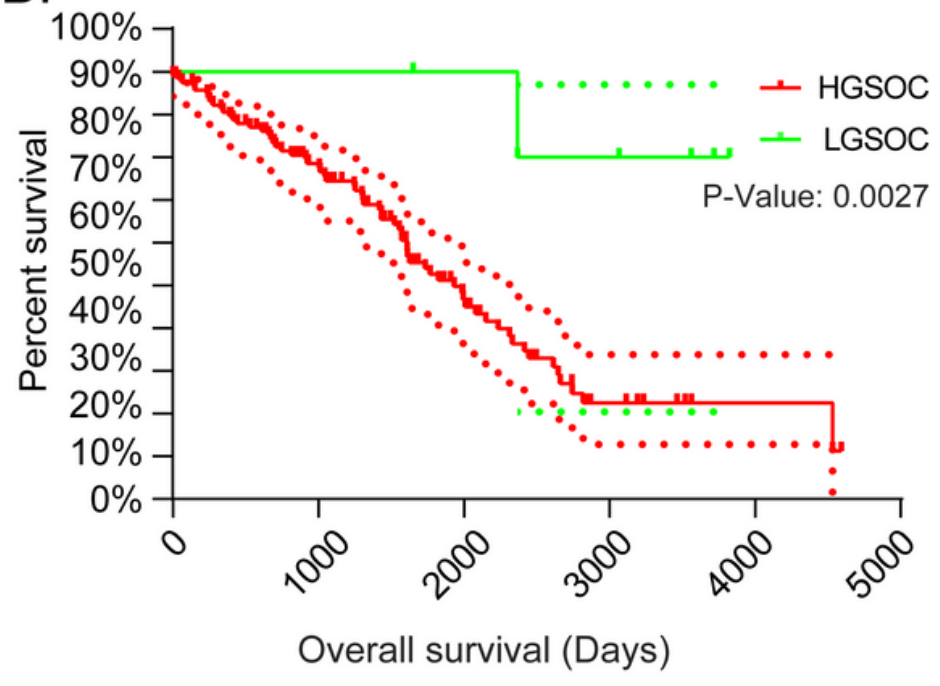

\section{Figure 1}

Progress-free survival(A) and overall survival(B) between high-grade and low-grade serous ovarian cancer through GSE151335. Progress-free survival and overall survival are predicted by Kaplan-Meier analysis. A log-rank $p$-value $<0.05$ was considered statistically significant. High-grade serous ovarian cancer(red line) sample number $=238$, low-grade serous ovarian cancer(green line) sample number $=6$ in GSE151335. 


\section{Overall Survival}

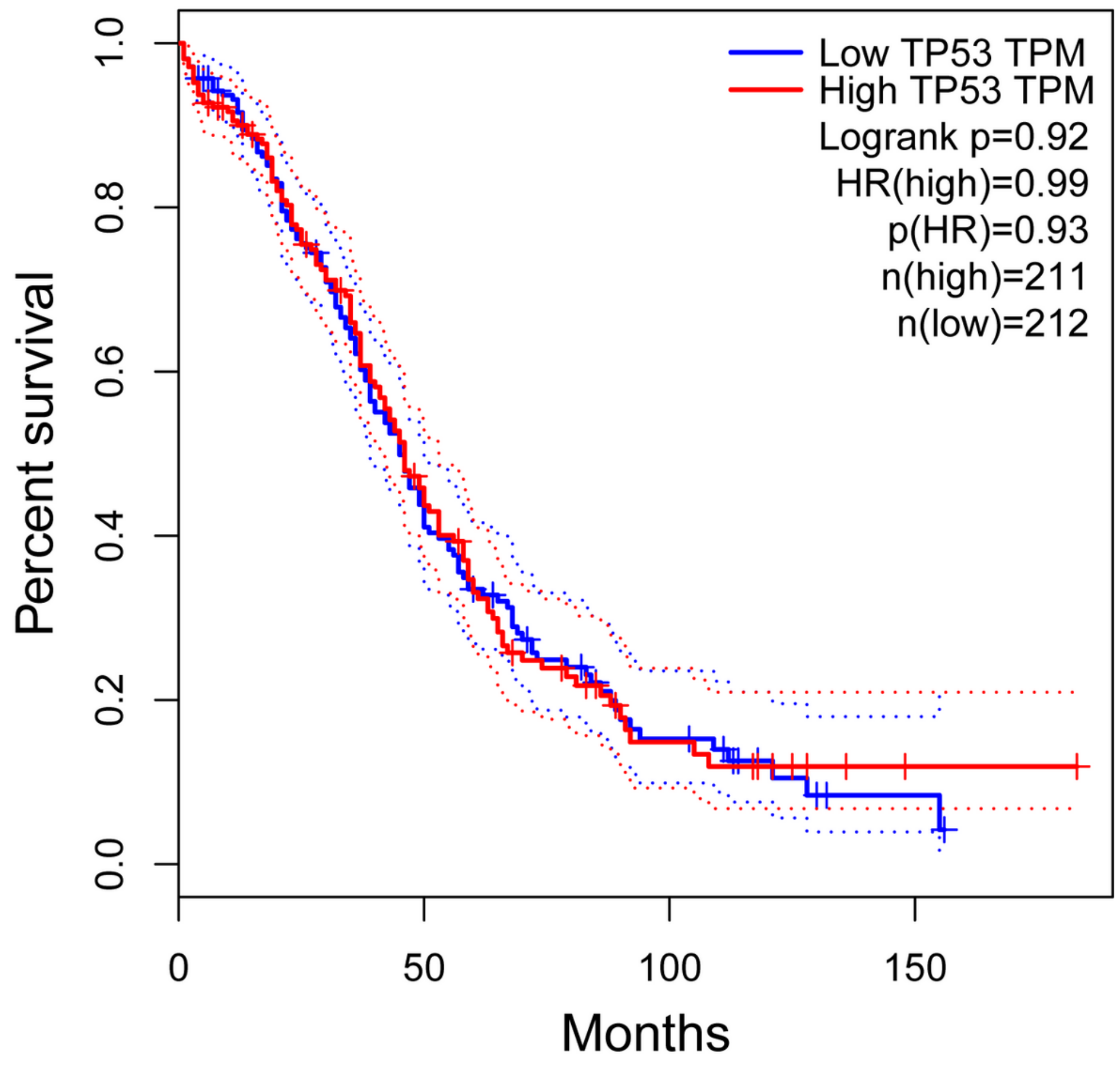

Figure 2

The prognostic information of TP53 in ovarian cancer. Overall survival is predicted by Kaplan-Meier analysis. A log-rank p-value $<0.05$ was considered statistically significant. The median seq expression level was set as the cutoff for the KM plot. Total patient number $=423$. The red line indicates high expression, and the blue indicates low expression. 

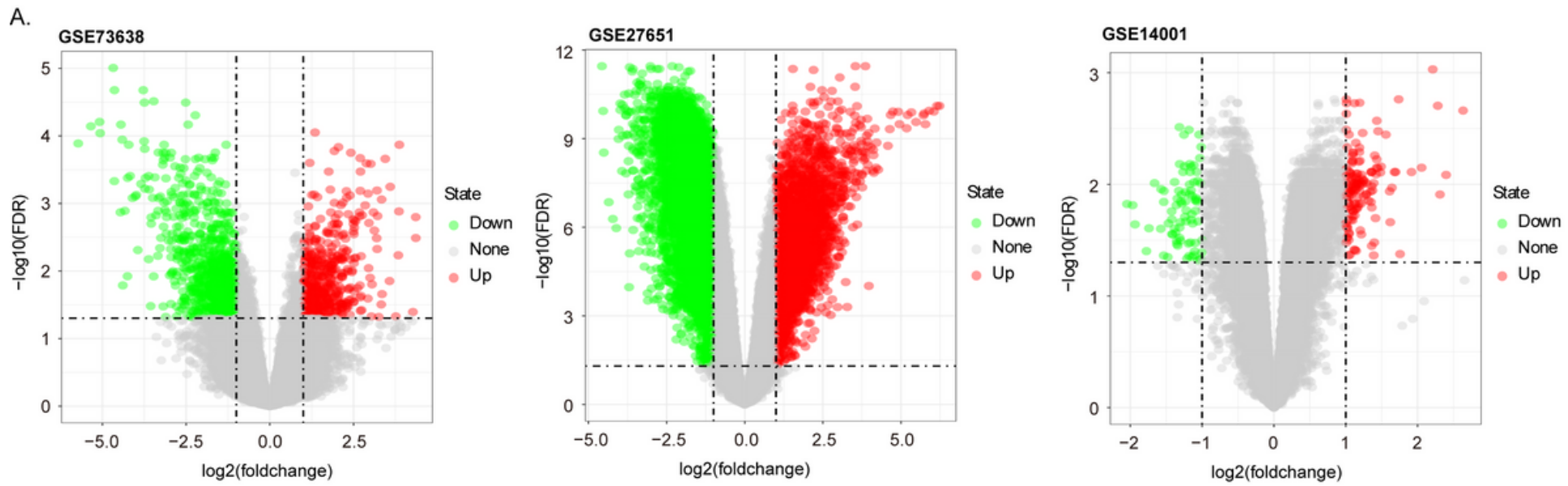

B.

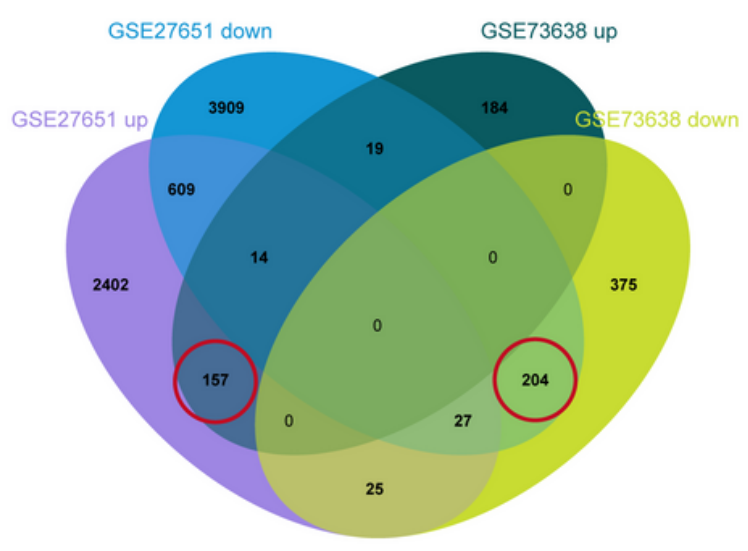

C.
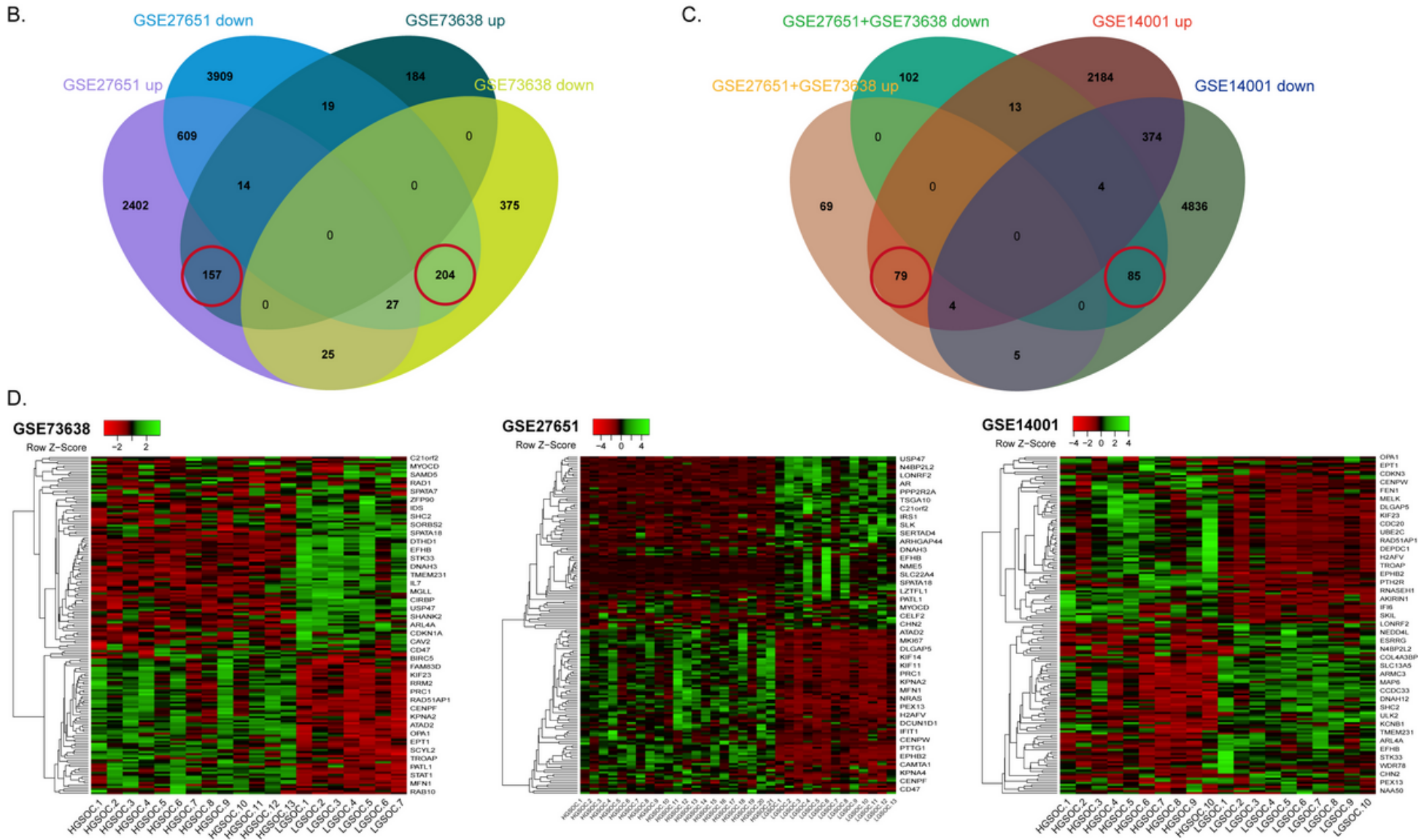

Figure 3

Screening of differential expressed genes between HGSOC and LGSOC. (A) Volcano plot of DEGs between HGSOC and LGSOC in each dataset. (B, C) Veen diagram of overlapping DEGs from GSE73638, GSE27651, and GSE14001. (D) Heatmap shows the distribution of DEGs data of each group extracted from GSE73638, GSE27651, and GSE14001. 
A.

mitotic cell cycle process

organelle fission

nuclear division

mitotic nuclear division

mitotic cell cycle

chromosome segregation

cell cycle process

sister chromatid segregation

C.

GSEA_GOBP in HGSOC

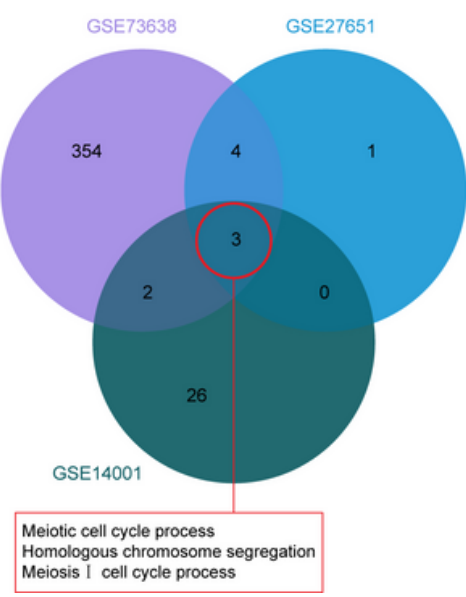

D.

GSE27651

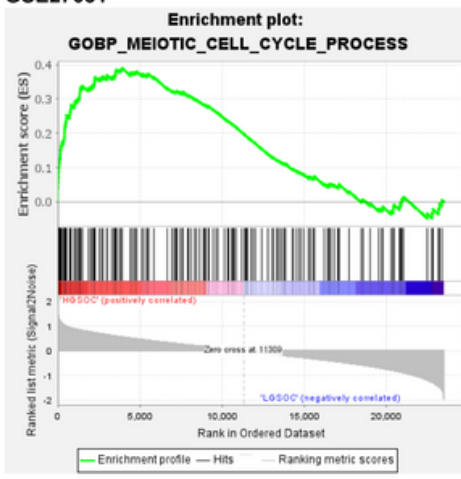

NES: 1.5526373 NOM p-value: 0.02636535
B.

enrichmentRatio

- 3

5
7

FDR

FDR
$9 e-09$

$9 e-09$
$6 e-09$

$-3 \mathrm{e}-09$

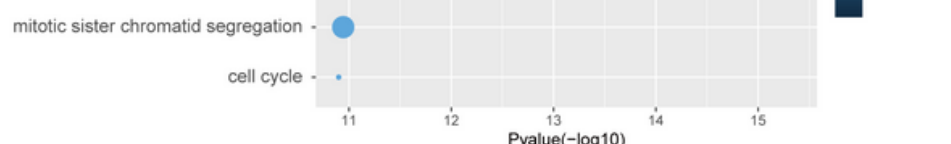

B. Pathway KEGG

Chronic myeloid leukemia

Oocyte meiosis

Colorectal cancer -

PI3K-Akt signaling pathway

Herpes simplex infection -

Insulin signaling pathway

Chemokine signaling pathway

Prostate cancer .
enrichmentRatio

-

9

7

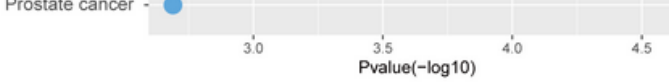

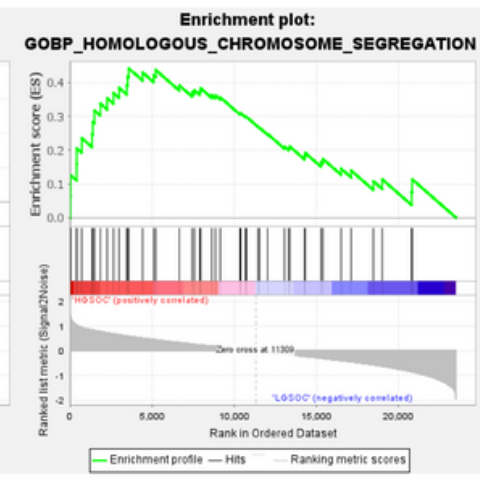

NES: 1.5094899 NOM p-value: 0.038817007

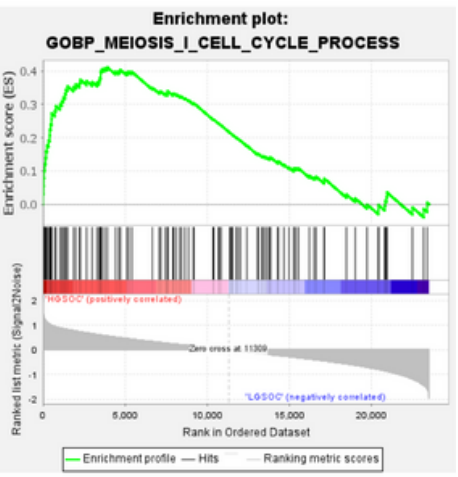

NES: 1.5558761 NOM p-value: 0.02247191

Figure 4

GO and KEGG analysis of the overlapping DEGs between HGSOC and LGSOC. (A) Top10 of GOBP enrichment via WebGestalt. (B) Top10 of KEGG pathway enrichment via WebGestalt. (C) Veen diagram of overlapping GOBP in HGSOC groups via GSEA. (D) The biological processes verified by the three GSEs. NES, normalized enrichment score; NOM p-value, Nominal p-value. 
A.
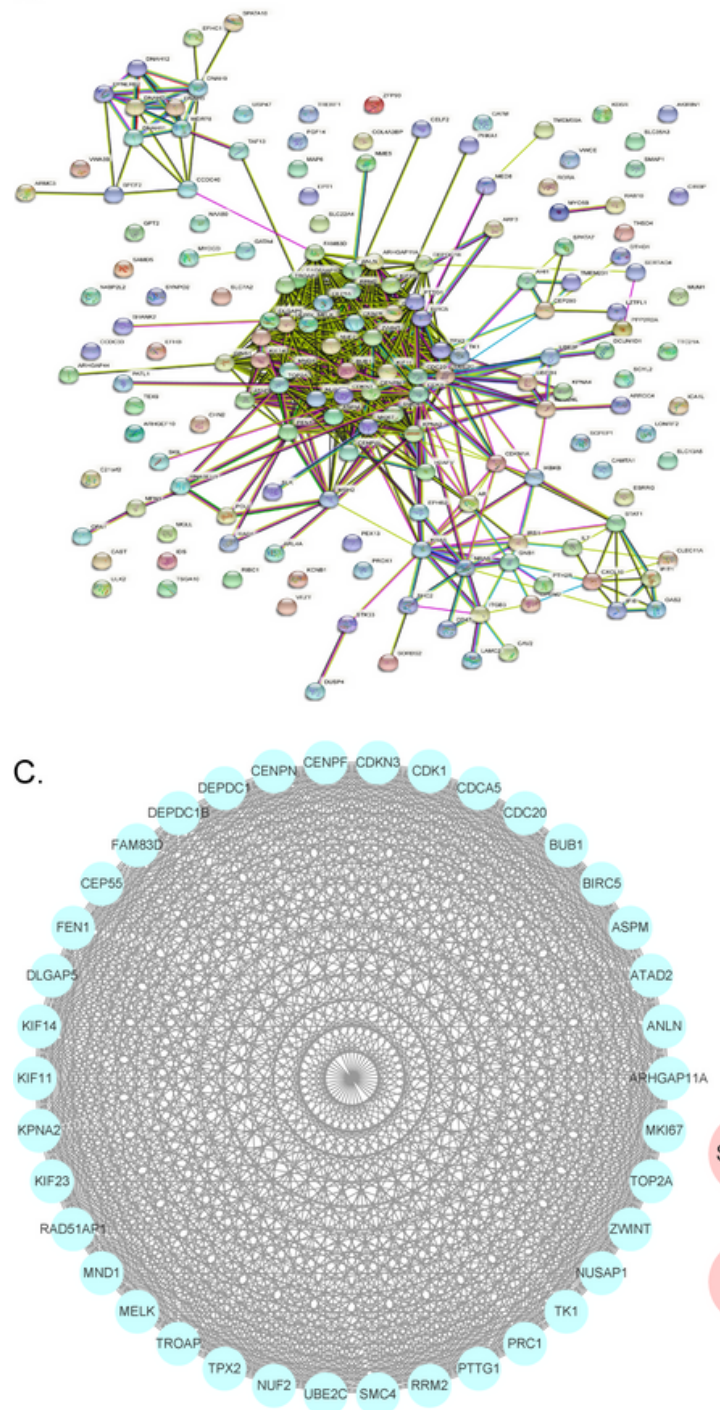

B.
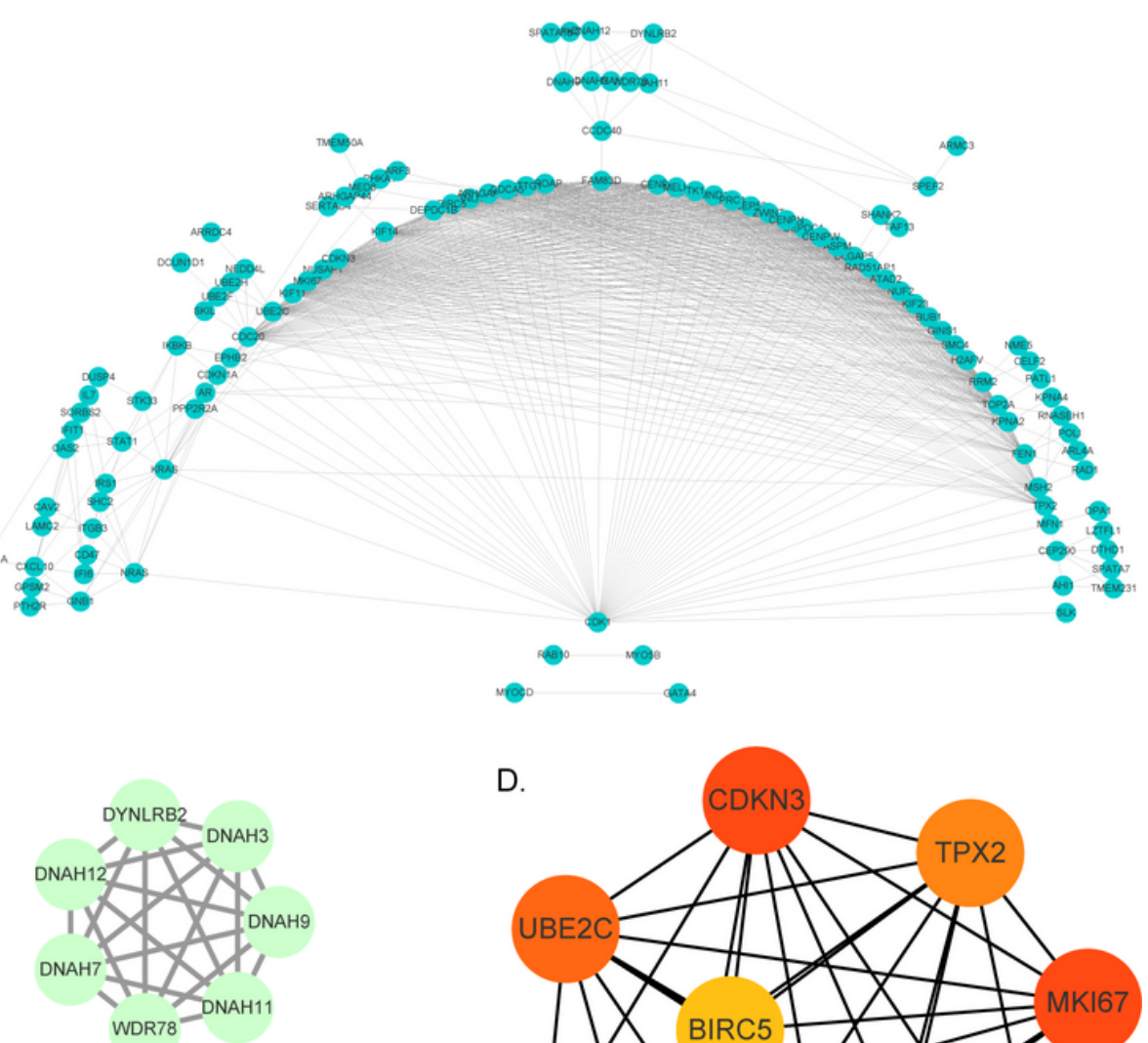

D.

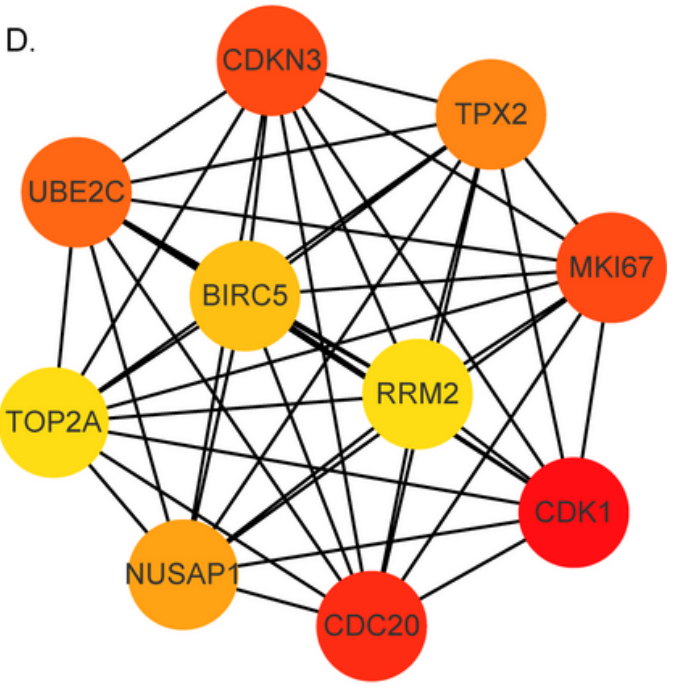

Figure 5

DEGs PPI network construction and module analysis. (A) PPI network via STRING. (B) PPI network via Cytoscape. (C) Module analysis by MCODE: degree cutoff $=2$, node score cutoff $=0.2, \mathrm{~K}$-Core $=2$, Max. Depth $=100$. (D) Hub genes analysis by cytoHubba (top 10 nodes ranked by Radiality algorithms). 


\section{A. BIRC5}

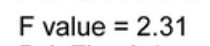

$\operatorname{Pr}(>\mathrm{F})=0.1$

num $(T)=426$

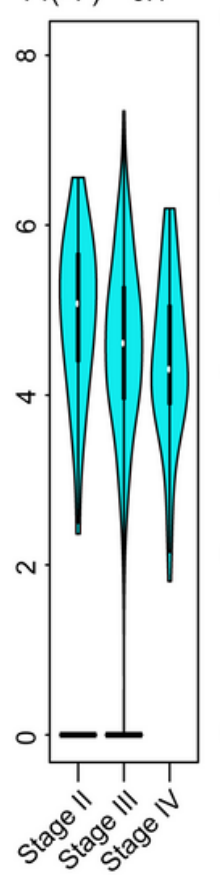

$\operatorname{num}(N)=88$

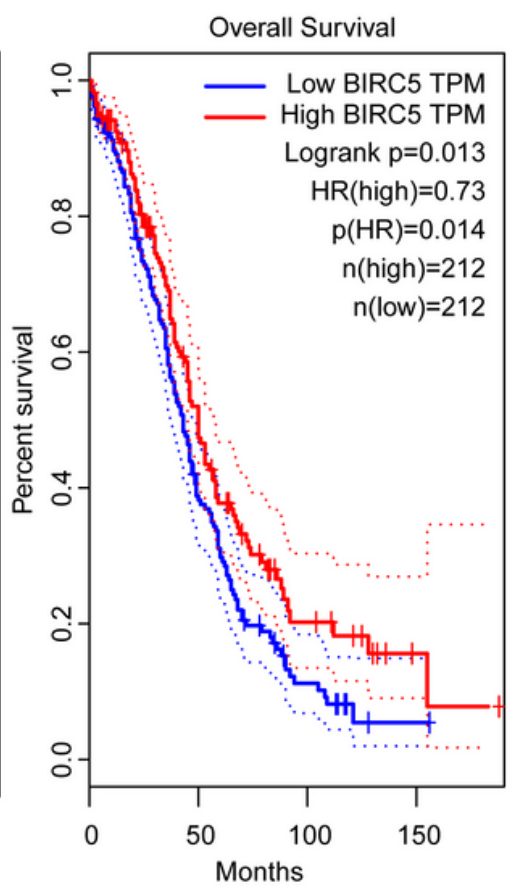

\section{B. RRM2}

$\mathrm{F}$ value $=3.72$

$\operatorname{Pr}(>\mathrm{F})=0.0251$

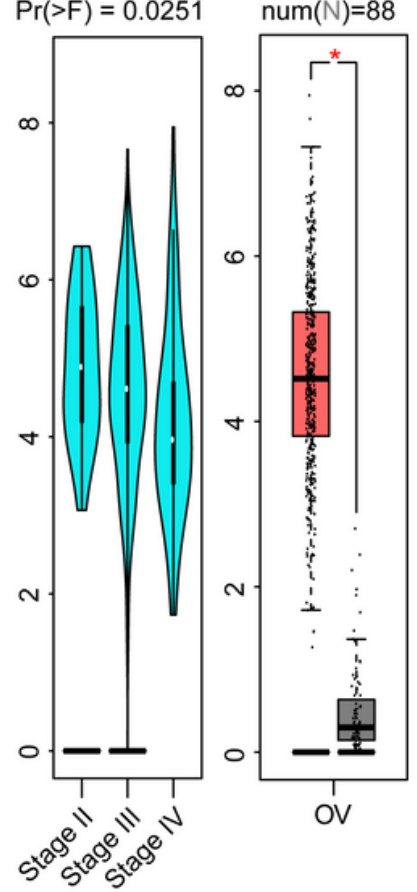

Overall Survival

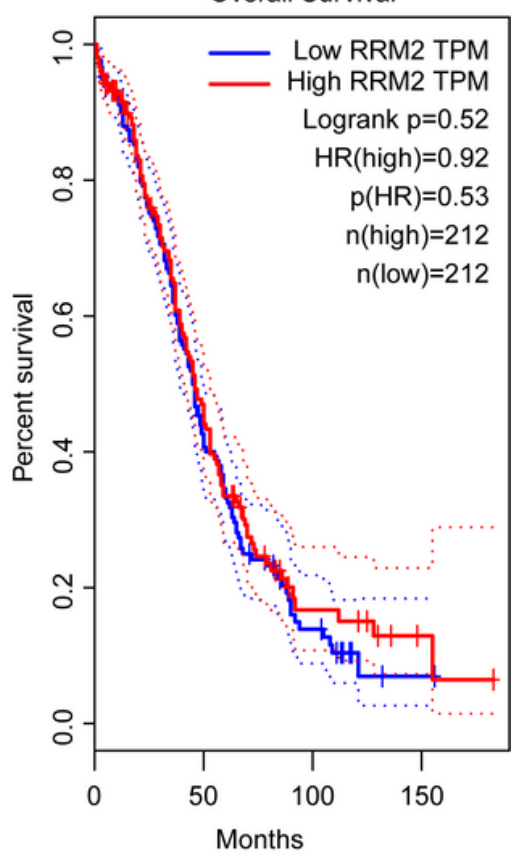

Figure 6

The stage, expression, and general survival information of hub genes BIRC5 (A) and RRM2 (B) in ovarian cancer (GEPIA). Ovarian cancer sample number $=426$ is in red, normal ovarian tissue number $=88$ is in gray. Overall survival is predicted by Kaplan-Meier analysis. A log-rank p-value $<0.05$ was considered statistically significant. The median seq expression level was set as the cutoff for the KM plot. Total patient number $=424$. The red line indicates high expression, and the blue indicates low expression. * $p$ $<0.05$, the statistically significant difference in expression. OV, ovarian cancer. 
A.
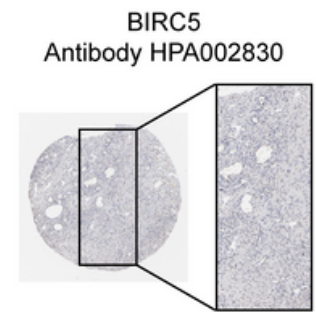

B.

Ovarian cancer

Not detected

Low

Medium
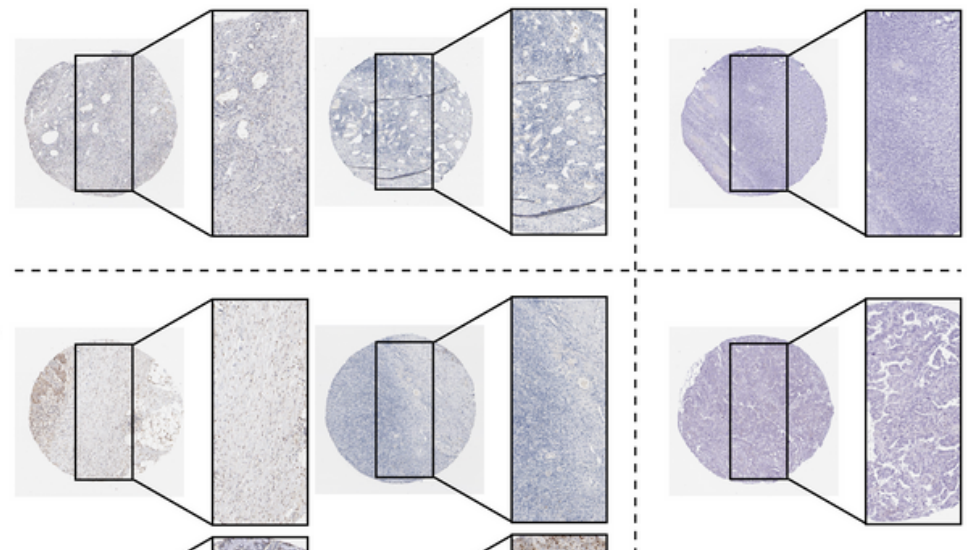

Antibody HPA056994

Antibody CAB004270

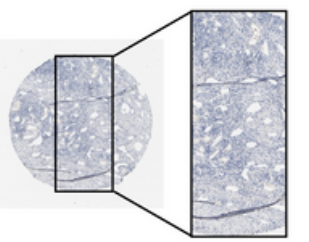

C.

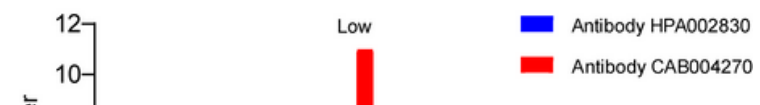

हू.

$6-$

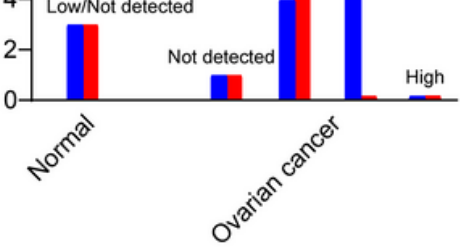

D.

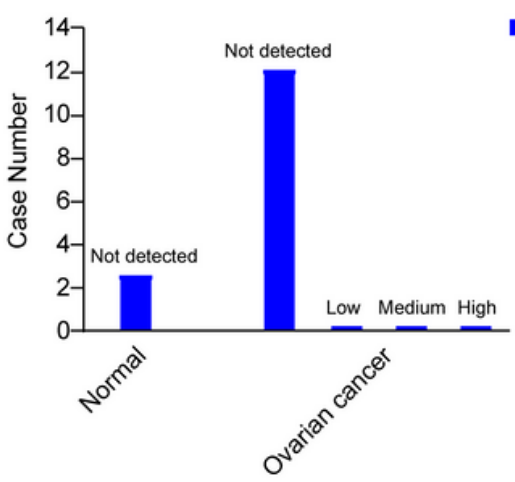

Figure 7

The IHC expression pattern of BIRC5 and RRM2 in normal ovarian tissue and ovarian cancer. (A) Normal ovarian tissue. (B) Representative IHC of ovarian cancer with not detected, low, and medium staining intensity. The black rectangle indicates a higher magnification of the indicated area in the picture. (C) Bar chart of IHC staining intensity of BIRC5 for ovarian cancer (total of 15 cases). (D) Bar chart of the IHC staining intensity of RRM2 for ovarian cancer (total of 15 cases). 
A. Allred score of BIRC5 口<3 口 $[3,5)$ 口 $[5,7)$ 口 $\geq 7$
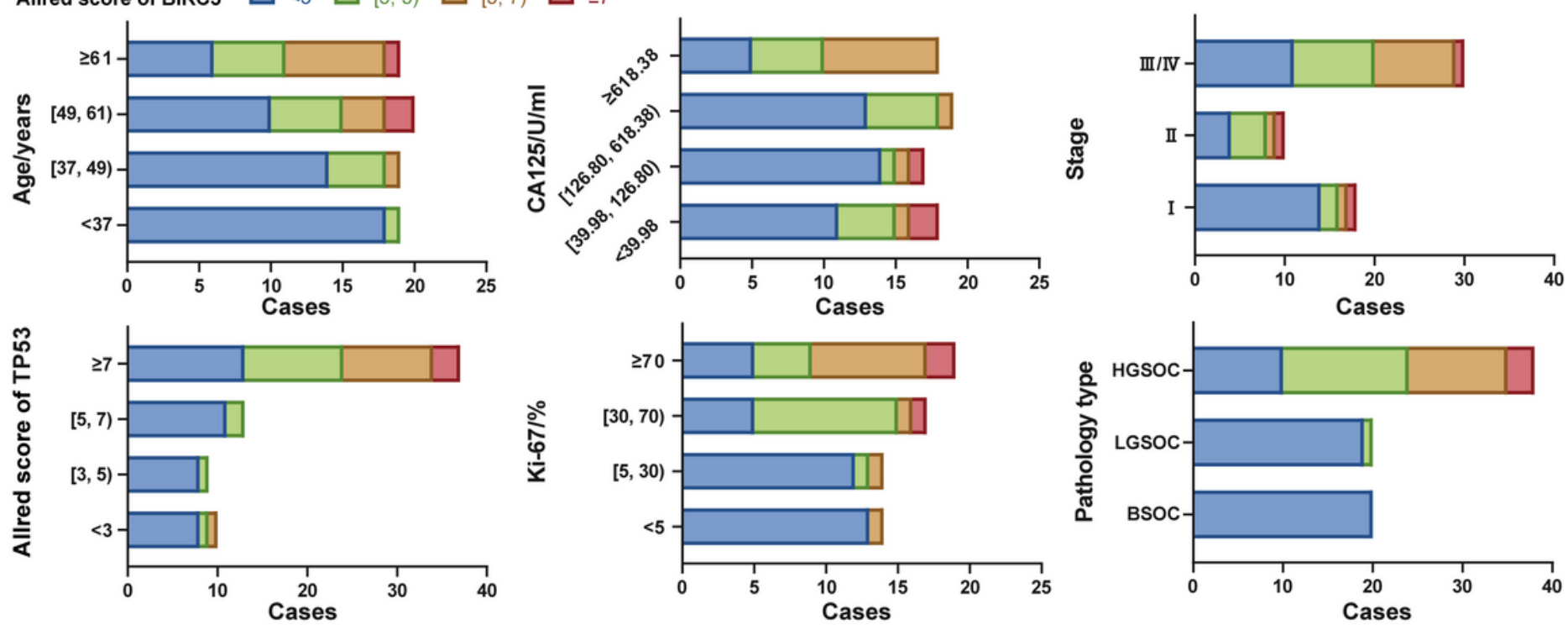

B.
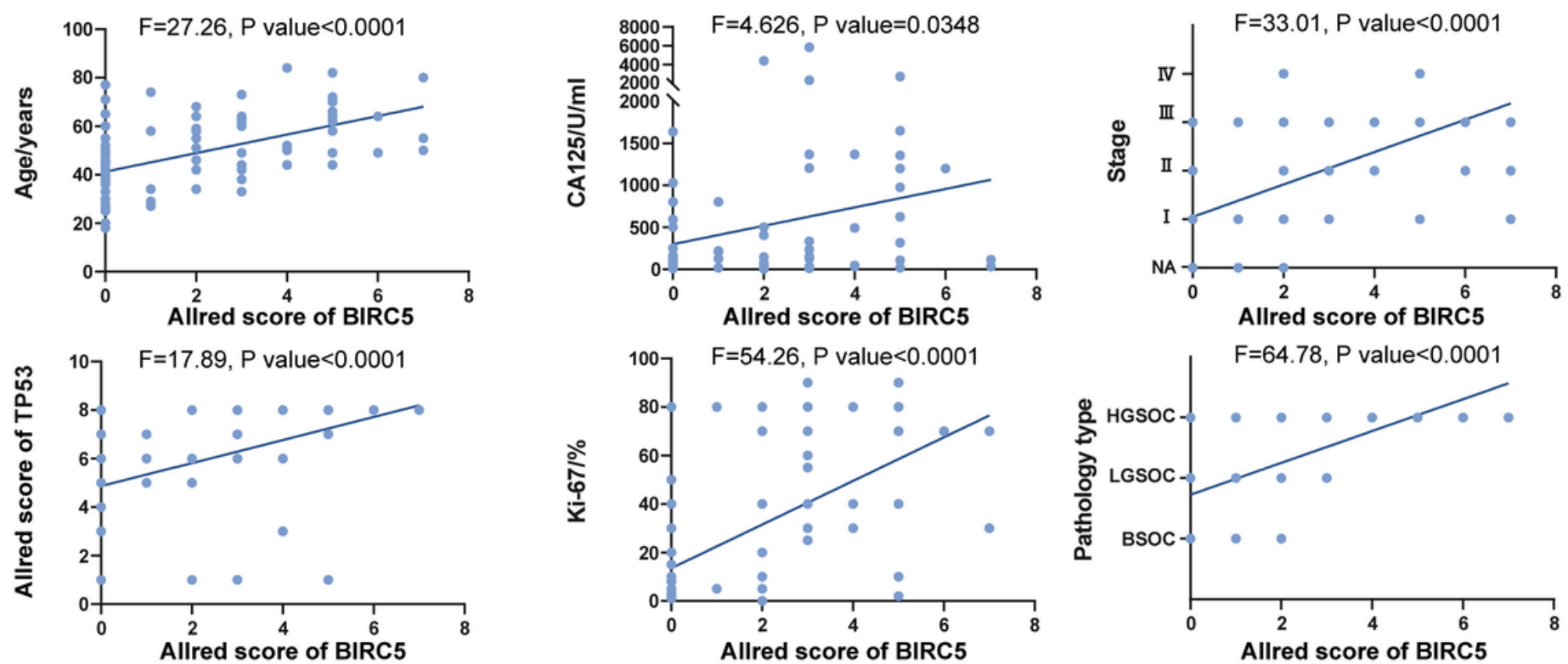

Figure 8

The correlation between BIRC5 expression and various clinicopathological parameters. (A) BIRC5 expression in different clinicopathological parameter groups. (B) Statistical correlation between BIRC5 expression and different clinicopathological parameters. 